\title{
3D Observation of Quasicrystal Alloy Using X-ray Differential Phase-Contrast Microscope with a Zone Plate
}

\author{
N. Watanabe ${ }^{1}$, S. Aoki ${ }^{2,1}$ \\ 1. Faculty of Pure and Applied Sciences, University of Tsukuba, Tsukuba, Ibaraki, 305-8573, Japan \\ 2. Comprehensive Research Organization for Science and Society, 1601 Kamitakatsu, Tsuchiura, Ibaraki, \\ 300-0811, Japan \\ *watanabe@bk.tsukuba.ac.jp
}

In the x-ray region above several $\mathrm{keV}$, phase-contrast is much higher than absorption contrast. We have been developing a differential phase-contrast microscope by using an $\mathrm{x}$-ray microscope with a zone plate and a Foucault knife-edge scanning filter [1]. A relatively quantitative differential phase image can be obtained by scanning a knife edge at the back focal plane of the objective zone plate. Using this microscope, differential phase-contrast imaging and phase tomography of biological samples could be successfully demonstrated [2].

Even in metal materials, phase-contrast in the x-ray region is much larger than absorption contrast. Therefore, in order to study the application of this microscope to metallic alloy, a quasicrystalline $\mathrm{Al}_{65} \mathrm{Cu}_{23} \mathrm{Fe}_{12}$ particle was observed. The particle was also observed by using absorption contrast and Zernike phase-contrast methods. The results of comparing the image quality of these images are reported in this paper.

The x-ray microscope was set at the beam line BL3C of the Photon Factory $(2.5 \mathrm{GeV}, 450 \mathrm{~mA})$. The optical system is shown in Fig. 1. The objective zone plate (NTT Advanced Technology Inc.) had the outermost zone width of $50 \mathrm{~nm}$ and the diameter of $330 \mu \mathrm{m}$. Monochromatic parallel x-rays of around 7 $\mathrm{keV}$ were incident onto a specimen and the transmitted x-rays were focused on a CCD camera (Hamamatsu Photonics C4742, pixel size $13 \mu \mathrm{m}$ ). The distance between a sample and the CCD camera was $3.6 \mathrm{~m}$ and the magnification ratio was 37. A gold wire of $250 \mu \mathrm{m}$ in diameter was used as a knifeedge. A differential phase image $I$ can be approximated by the formula $I \approx 2 \pi S\left(I_{+}-I_{-}\right) /\left(I_{+}+I_{-}\right)$, where $I_{+}$ and $I$ - are recorded images with opposite scan directions of the knife-edge and $S$ is the spatial frequency corresponding to the scan width of the knife-edge [2]. The scan width was $\pm 5 \mu \mathrm{m}$ centering on the back focal point of the zone plate in this experiment. Computed tomography (CT) images were calculated from 360 differential phase images from the different angles of view at angular interval of 1.0 degree. A Zernike phase-contrast image was observed by replacing the knife edge with a Zernike phase plate. A 3$\mu \mathrm{m}$-thick aluminum foil with a pinhole of $6 \mu \mathrm{m}$ in diameter was used as the phase plate.

A quasicrystalline $\mathrm{Al}_{65} \mathrm{Cu}_{23} \mathrm{Fe}_{12}$ particle (SIGMA-ALDRICH Corp) was adhered to a glass capillary and mounted on a rotating sample stage. The X-ray images are shown in Fig. 2. Figure 2(a) is the absorption image and Fig. 2(b) is the corresponding CT section image at $7.2 \mathrm{keV}$. Figure 2(c) is the Zernike phasecontrast image and Fig. 2(d) is the corresponding CT section image at $7.0 \mathrm{keV}$. Figure 2(e) is the differential phase image and Fig. 2(f) and 2(g) are the corresponding CT images at $7.2 \mathrm{keV}$.

In all images, existence of voids can be confirmed. These voids are thought to be related to the brittleness of particles. The voids could be observed with good contrast in the differential phase-contrast method. The Zernike phase-contrast image also has good contrast. But the CT image from the Zernike 
phase-contrast images has serious artifacts because of the lack of the phase linearity. These results show that the differential phase-contrast method is effective for 3D observation of internal voids in such an alloy sample.

\section{References:}

[1] N. Watanabe et al, J. Phys.: Conf. Ser. 463 (2013), 012011.

[2] N. Watanabe, et al, AIP Conf. Proc. 1696 (2016), 020044.

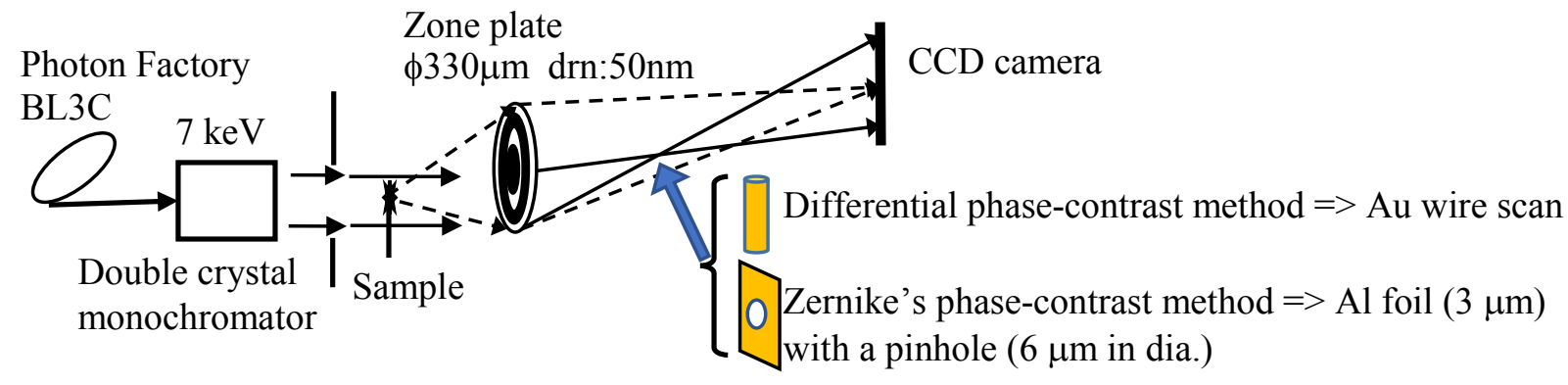

Figure. 1. Optical system of x-ray differential phase-contrast microscope.
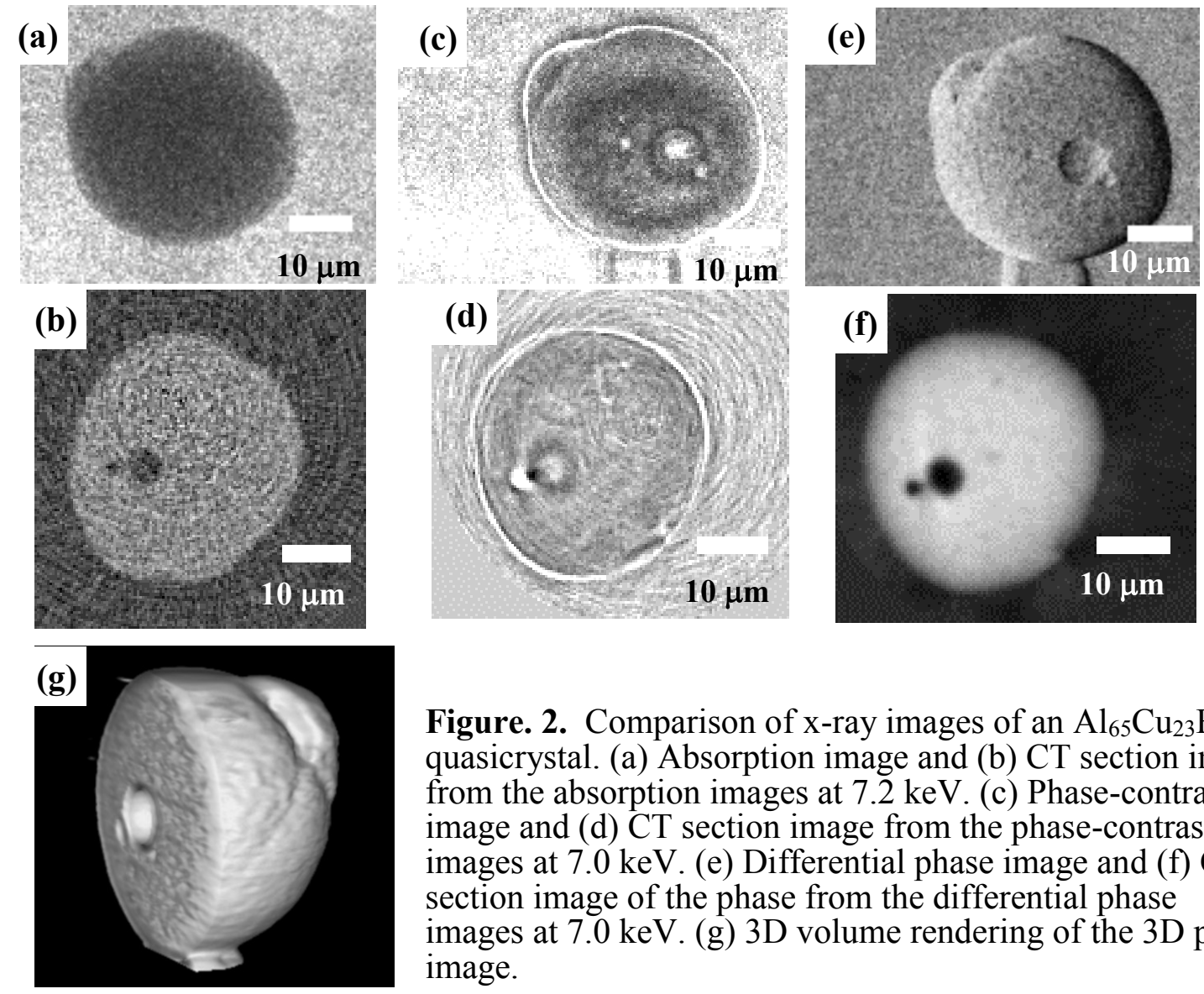

Figure. 2. Comparison of $x$-ray images of an $\mathrm{Al}_{65} \mathrm{Cu}_{23} \mathrm{Fe}_{12}$ quasicrystal. (a) Absorption image and (b) CT section image from the absorption images at $7.2 \mathrm{keV}$. (c) Phase-contrast image and (d) CT section image from the phase-contrast images at $7.0 \mathrm{keV}$. (e) Differential phase image and (f) CT section image of the phase from the differential phase images at $7.0 \mathrm{keV}$. (g) 3D volume rendering of the 3D phase image. 